\title{
THE ANTIAUTOMORPHISM OF THE STEENROD ALGEBRA
}

\author{
DONALD M. DAVIS
}

Abstract. Combinatorial techniques are used to obtain some formulas for the canonical antiautomorphism of the Steenrod algebra.

In this note we shall prove some nice formulas involving the canonical antiautomorphism $\chi$ of the $\bmod p$ Steenrod algebra [1].

THEOREM 1. $\quad \chi\left(\mathscr{P}^{p^{n-1}+\cdots+p+1}\right)=(-1)^{n} \mathscr{P}^{n-1} \cdots \mathscr{P}^{p} \mathscr{P}^{1}$.

THEOREM 2. For $n \geqq k, \chi\left(S q^{2^{n}-k}\right)=S q^{2^{n-1}} \cdots S q^{2^{k-1}}\left(\chi\left(S q^{2^{k-1}-k}\right)\right)$.

$\chi\left(S q^{2^{k-1}-k}\right)=S q^{2^{k-2}} \chi\left(S q^{2^{k-2}-k}\right)+S q^{2^{k-2}-1} S q^{2^{k-3}-1} \cdots S q^{3} S q^{1}$.

I wish to thank Mark Mahowald, Sholom Rosen, and Greg Burnham for helpful suggestions.

Let $S(i)$ denote the sum of all Milnor basis elements [1] of the form $\mathscr{P}^{R}$ in dimension $i$. In $[1$, Corollary 6$]$ is it shown that $\chi\left(\mathscr{P}^{i}\right)=(-1)^{i} S(2 i(p-1))$. Thus Theorem 1 will follow by induction once we have shown that $\mathscr{P}^{p^{n-1}} \cdot S\left(2\left(p^{n-1}-1\right)\right)=S\left(2\left(p^{n}-1\right)\right)$. Indeed we shall prove

Proposition.

$$
\mathscr{P}^{m} \cdot S(l)=\sum_{R}\left(\begin{array}{c}
\sum p^{i} r_{i} \\
p m
\end{array}\right) \mathscr{P}^{R},
$$

where the sum is taken over all sequences $R=\left(r_{1}, \cdots\right)$ having $\sum 2\left(p^{i}-1\right) r_{i}=$ $l+2 m(p-1)$.

Then we need merely to note that if $\sum 2\left(p^{i}-1\right) r_{i}=2\left(p^{n}-1\right)$, then $\sum p^{i} r_{i}=$ $p^{n}-1+\sum r_{i}$ and $1 \leqq \sum r_{i} \leqq 2\left(p^{n}-1\right) / 2(p-1)$, and hence $p^{n} \leqq \sum p^{i} r_{i} \leqq$ $p+\cdots+p^{n}$, so that

$$
\left(\begin{array}{c}
\sum p^{i} r_{i} \\
p^{n}
\end{array}\right) \equiv 1(\bmod p)
$$

Proof of Proposition. The product contains a term

$$
\prod\left(\begin{array}{l}
r_{i} \\
a_{i}
\end{array}\right) \mathscr{P}^{\left(r_{1}, \ldots\right)}
$$

Received by the editors June 18, 1973 and, in revised form, September 7, 1973. AMS (MOS) subject classifications (1970). Primary 55G10; Secondary 05A15. Key words and phrases. Steenrod algebra, antiautomorphism, Milnor basis.

(c) American Mathematical Society 1974 
for each Milnor matrix

$$
\begin{array}{|cccc}
\hline & r_{1}-a_{1} & r_{2}-a_{2} & \cdots \\
a_{1} & a_{2} & a_{3} & \cdots
\end{array}
$$

such that $\sum p^{i-1} a_{i}=m$. In summing these, it is useful to note that if $s_{1}, \cdots$ is a finite sequence of positive integers and $B$ ranges over all sequences $b_{1}, \cdots$ such that $0 \leqq b_{i} \leqq s_{i}$ and $\sum b_{i}=k$, then

$$
\sum_{B} \prod_{i}\left(\begin{array}{c}
s_{i} \\
b_{i}
\end{array}\right)=\left(\begin{array}{c}
\sum s_{i} \\
k
\end{array}\right)
$$

This is proved by comparing coefficients of $x^{k}$ in $\Pi(1+x)^{s_{i}}=(1+x)^{\Sigma s_{i}}$. Also we shall make use of the well-known facts

$$
\left(\begin{array}{l}
r \\
a
\end{array}\right) \equiv\left(\begin{array}{c}
p^{i} r \\
p^{i} a
\end{array}\right) \bmod p, \quad \text { and } \quad\left(\begin{array}{c}
p^{i} r \\
b
\end{array}\right) \equiv 0 \bmod p
$$

if $b$ is not divisible by $p^{i}$. These are proved by comparing coefficients of $x^{p^{i} a}$ (respectively $\left.x^{b}\right)$ in $(1+x)^{p^{i} r} \equiv\left(1+x^{p^{i}}\right)^{r}$.

Thus we have

$$
\begin{aligned}
\mathscr{P}^{m} \cdot S(l) & =\sum_{R} \sum_{A} \prod_{i}\left(\begin{array}{c}
r_{i} \\
a_{i}
\end{array}\right) \mathscr{P} R \equiv \sum_{R} \sum_{A} \prod_{i}\left(\begin{array}{c}
p^{i} r_{i} \\
p^{i} a_{i}
\end{array}\right) \mathscr{P} R \\
& \equiv \sum_{R} \sum_{B} \prod_{i}\left(\begin{array}{c}
p^{i} r_{i} \\
b_{i}
\end{array}\right) \mathscr{P} R=\sum_{R}\left(\begin{array}{c}
p^{i} r_{i} \\
p m
\end{array}\right) \mathscr{P} R
\end{aligned}
$$

Here $R$ ranges over sequences $\left(r_{1}, \cdots\right)$ having $\sum 2\left(p^{i}-1\right) r_{i}=l+2 m(p-1)$, $A$ ranges over sequences $\left(a_{1}, \cdots\right)$ having $\sum p^{i-1} a_{i}=m$, and $B$ ranges over sequences $\left(b_{1}, \cdots\right)$ having $\sum b_{i}=p m$.

Theorem 2 follows by similar techniques using the following lemmas, which are easily proved by induction.

LEMmA 1. If $\sum\left(2^{i}-1\right) r_{i}=2^{n}-k$ with $n \geqq k$, then $\sum r_{i} \geqq k$. If $n=k-1$, the above is true except for the case when all $r_{i}=1$.

LeMmA 2. $S q^{2^{k}-1} S q^{2^{k-1}-1} \cdots S q^{3} S q^{1}$ equals the Milnor basis element having 1 in the first $k$ components.

\section{REFERENCE}

1. J. Milnor, The Steenrod algebra and its dual, Ann. of Math. (2) 67 (1958), 150-171. MR 20 \#6092.

Department of Mathematics, Northwestern University, Evanston, Illinois 60201 